\title{
Applications of Carbohydrate-Gold Nanoparticles for Volumetric Flow Measurements Using an Opto-Acoustic Technique
}

\author{
Chan-Yi Yang ${ }^{\mathrm{a}}$ ( 楊正義), Chen-Wei Weib (魏振偉), Yen-Ping Lin ${ }^{\mathrm{a}}$ (林燕萍),

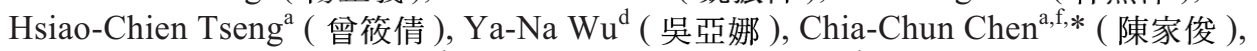 \\ Dar-Bin Shieh ${ }^{\mathrm{c}, \mathrm{d}, \mathrm{e}, *}$ ( 謝達斌) and Pai-Chi Li ${ }^{\mathrm{b}, *}$ ( 李百祺 ) \\ ${ }^{a}$ Department of Chemistry, National Taiwan Normal University, Taipei 116, Taiwan, R.O.C. \\ ${ }^{\mathrm{b}}$ Department of Electrical Engineering, National Taiwan University, Taipei 106, Taiwan, R.O.C. \\ ${ }^{\mathrm{c}}$ Institute of Oral Medicine, National Cheng-Kung University, Tainan 701, Taiwan, R.O.C. \\ ${ }^{\mathrm{d}}$ Institute of Molecular Medicine, National Cheng-Kung University, Tainan 701, Taiwan, R.O.C. \\ ${ }^{\mathrm{e}}$ Institute of Basic Medicine, National Cheng-Kung University, Tainan 701, Taiwan, R.O.C. \\ ${ }^{\mathrm{f}}$ Institute of Atomic and Molecular Sciences, Academia Sinica, Taipei 106, Taiwan, R.O.C.
}

Gold nanoparticles with carbohydrate ligands attached on their surface have been synthesized and characterized with various techniques. The new nanoparticle conjugates have shown great potentials as a contrast agent for opto-acoustic imaging. Hemocompatibility measurements of human blood for the carbohydrate-gold nanoparticles have shown that the conjugates are feasible for in vivo testing. Preliminary quantitative flow measurements using the conjugates were also studied in this work based on the indicator-dilution theory. In vitro phantom experiments were designed and conducted, and results were discussed.

Keywords: Carbohydrate-AuNPs; Opto-acoustic imaging; Time-intensity curve.

\section{INTRODUCTION}

Gold nanoparticles coupled with biomolecules have been long used for applications in biological labeling, imaging and assays. ${ }^{1}$ In immunoassay systems, many current diagnostic kits have been developed on the basis of interactions between antibody conjugated gold nanoparticles and their antigens. ${ }^{2}$ Of late, gold nanoparticles coupling with DNA single or double strains have shown great potentials for detecting unknown gene sequences as well as for gene delivery purposes. ${ }^{3}$ Also, the uses of the nanoparticles for biological and medical imaging have been also widely demonstrated because of the excellent contrast of gold nanoparticles under an electron microscope. ${ }^{4}$

There are several technical advantages of using gold nanoparticles for biological labeling, imaging and assays. First, the sizes of gold nanoparticles are comparable to those of small molecules, short peptides and DNA sequences. Therefore, the nanoparticle can be easily coupled with those materials to generate new nanoparticle conjugates with dual or multiple biological functions. For exam- ple, the gold nanoparticles coupled with carbohydrate molecules have shown that they can be applied to identify the binding site of a specific protein on the pili of E. coli. ${ }^{5} \mathrm{Sec}-$ ond, a single nanoparticle with a large surface volume ratio is ready for the covalent attachment of multiple ligands, which may generate a strong binding affinity between the nanoparticle conjugates and the specific binding target. ${ }^{6}$ Third, the gold nanoparticles exhibit an intense color in the visible region for spectroscopic detection and also offer great contrast for imaging so that they are feasible for application for many new biological labeling and imaging purposes. Finally, gold nanoparticles are biological compatible materials so that they are safe for use in biological detection or in imaging.

Up to now, relatively few examples have demonstrated the uses of gold nanoparticles conjugates as contrast agents. The contrast agent has been used for signal enhancement in many biomedical imaging modalities. In ultrasound, for example, microbubble based contrast agents are used to enhance the acoustic backscattered intensity of blood and thereby assist the assessment of blood flow pa-

\footnotetext{
* Corresponding author. Tel: (886)-02-89316151; Fax: (886)-02-89316363; E-mail: cjchen@cc.ntnu.edu.tw; paichi@cc.ee.ntu.edu.tw; dshieh@mail.ncku.edu.tw
} 
rameters. In addition to signal enhancement, several contrast-specific imaging techniques based on the indicator-dilution theory have also been proposed. ${ }^{7}$ The indicator-dilution theory provides a mathematical model for estimating hemodynamic parameters using changes in the signal intensity as a function of time (i.e., the time-intensity curve, TIC). A linear time-invariant system model is generally assumed such that the TIC can be used to represent the transfer function of the dilution process. ${ }^{8}$ Based on the dilution transfer function, blood flow information can be subsequently estimated. In this study, we will investigate the feasibility of the indicator-dilution based flow measurement techniques for a different imaging modality: opto-acoustic (OA) imaging. OA imaging is a new imaging technique and has been used to image the optical properties of biological tissues. In biomedical applications, OA imaging takes advantage of high optical contrast and low acoustical scattering, and hence represents a promising tool for breast tumor prediction, epidermal melanin measurement, and monitoring of oxygenation in blood vessels. Although OA imaging has demonstrated its biomedical potential, it is still used mostly as a morphological imaging method. To extend OA imaging to functional and molecular levels, we have synthesized different types of carbohydrates attached on gold nanoparticles to form new types of carbohydrate-gold nanoparticles (carbohydrate-AuNPs). We use those conjugates as the OA contrast agent. It is the main purpose of this study to test the hypothesis that carbohydrate-AuNPs can be used to develop quantitative flow measurement techniques based on the indicator-dilution theory. Hemocompatibility of human blood for the carbohydrate-AuNPs are also studied in this work. Specifically, in vitro phantom experiments are designed and conducted, and results and future projects are discussed.

\section{EXPERIMENTAL SECTIONS}

\section{Preparation of Carbohydrate-Gold Nanoparticles}

Various carbohydrate-AuNP (approx. $40 \mathrm{~nm}$ ) as described in Fig. 1 were synthesized following similar methods as previously reported. ${ }^{5} \mathrm{An}^{\mathrm{HAuCl}} 4$ aqueous solution $\left(3 \mathrm{~mL}, 15 \mathrm{mmolL}^{-1}\right)$ was added to a toluene solution $(3 \mathrm{~mL}$, $\left.35 \mathrm{mmolL}^{-1}\right)$ with tetraoctylammonium bromide $(2 \mathrm{mg})$ at room temperature. After stirring for $1 \mathrm{~min}$, the organic layer was separated out from the aqueous solution. To sodium borohydride ( $3 \mathrm{mg}$ ) was slowly added to the aqueous solution to a freshly prepared methanol solution of differ- ent carbohydrates such as mannose, glucose, galactose (5 $\mathrm{mg}$ ) with vigorous stirring. After stirring for $0.5 \mathrm{~h}$, gold nanoparticles were precipitated by centrifugation and then washed with methanol $(20 \mathrm{~mL} \times 3)$. The carbohydrate encapsulated nanoparticles were dried under vacuum overnight.

\section{Characterization of Carbohydrate-Gold Nanoparticles Transmission electron microscopy}

The diameter of the carbohydrate-AuNPs were determined by transmission electron microscopy (TEM). A small drop of the conjugate suspension was placed on a carbon-coated copper grid, and excess solution was evaporated away by a fluorescent lamp. The grid was subsequently dried in air and imaged on a Hitachi H-7100 transmission electron microscope. The sizes of the mannosegold nanoparticles ( $m$-AuNPs) were determined by the TEM image analyses.

\section{UV-vis absorption spectroscopy}

A typical $m$-AuNPs solution for the stability study was prepared by dissolving $3 \mathrm{mg} m$-AuNPs in $10 \mathrm{~mL}$ water (O.D value 0.704$)$. The above solution was mixed with different media in a $v / v=1 / 1$ ratio. The measurements were recorded on UV-near-IR spectrometers (Perkin Elmer, $\lambda-900$ and HP-8453).

\section{Hemocompatibility of Human Blood for the Carbohy- drate-Gold Nanoparticles}

Because the nanoparticles were designed for the applications as a blood flow contrast agent in circulation, the hemocompatibility issue must be addressed. A hemolysis assay was performed using human whole blood from a healthy donor with permission following guidelines for studies using human specimens. Different concentrations of the $m$-AuNPs were added to $1 \mathrm{~mL}$ human whole blood

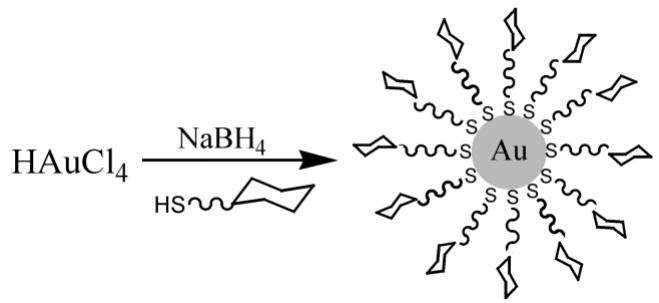

Fig. 1. Synthesis of the carbohydrate-gold nanoparticles. Formation of carbohydrate-AuNPs coated with various carbohydrates such as mannose, glucose and galactose by reduction of $\mathrm{Au}^{\mathrm{III}}$ compounds. 
stored in the vacutainer (BD Inc., USA) containing 120 IU sodium heparin in a serial 2-fold dilution from the original concentration of $9.7 \times 10^{-9} \mathrm{M}$ nanoparticles to a minimal concentration of $1.89 \times 10^{-11} \mathrm{M}$. The tubes were gently mixed in a rotary shaker and then incubated for 4 hours at $37^{\circ} \mathrm{C}$. The serum in the supernatant was collected after centrifugation under $300 \times \mathrm{g}$ for $10 \mathrm{~min}$. The serum was further centrifuged under $20000 \times \mathrm{g}$ to remove the nanoparticles, and the supernatant was analyzed for the presence of the hemoglobin by spectrophotomertic absorption $(454 / 528 \mathrm{~nm})$ in a Coulter Gen-S analyzer (Beckman Inc, USA).

\section{Opto-Acoustic Measurements}

Measuring the TIC is schematically shown in Fig. 2. The system consisted of a precision translation stage (CSR200, CSIM, Taiwan), a laser system, and an ultrasonic receiving system. A frequency-doubled Nd:YAG laser (LS-2132U, LOTIS TII) operating at $532 \mathrm{~nm}$ with a pulse duration of $5 \mathrm{~ns}$ was used for optical illumination. The optical energy of the laser pulse was $7 \mathrm{~mJ}$ and the pulse repetition rate was $15-\mathrm{Hz}$. An ultrasonic single crystal transducer (V-381, Panametrics) with a center frequency of $3.5 \mathrm{MHz}$ and a focal depth of $7 \mathrm{~cm}$ was employed to detect the OA waves. The acoustic waveforms were amplified by an amplifier (5800PR, Panametrics) and then recorded by a data acquisition card (CompuScope 12100, Gage) at a 100$\mathrm{MHz}$ sampling rate. The acquired data were stored in a personal computer for subsequent data analysis.

For OA TIC measurements, gold nanoparticles with an average diameter of $40 \mathrm{~nm}$ were injected into a vessel in a PVA phantom with a diameter of $5 \mathrm{~mm}$. The particles then flew through a mixing chamber of $30 \mathrm{~mL}$, which was used to simulate the situations where the injection site is far

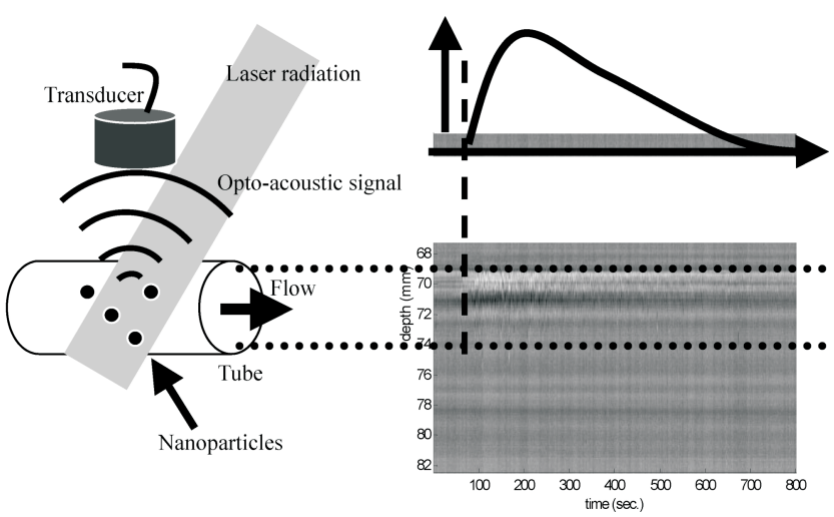

Fig. 2. Schematic diagram for OA TIC measurements. away from the measurement site. ${ }^{7 \mathrm{c}}$ In front of the injection site, a damper was used to stabilize the flow. Laser irradiation was from the top of the vessel and the acoustic detection was from the side. To prevent recirculation of the nanoparticles back in the phantom, the water only passed through the model once.

\section{RESULTS AND DISCUSSION}

\section{Properties and Characterization of Carbohydrate- Gold Nanoparticles}

The spherical shape of $m$-AuNPs was observed in a typical TEM image (Fig. 3). Individual nanoparticles were found in the images without any aggregation. The result indicates that the $m$-AuNPs are quite water-soluble and dispersed well in the water-based solution. Both UV-visible spectra of the gold nanoparticles before and after coupling with carbohydrate showed a clear plasmon band of $\lambda \approx 520$ nm (Fig. 4). ${ }^{9}$ No red shift or intensity decrease of the band

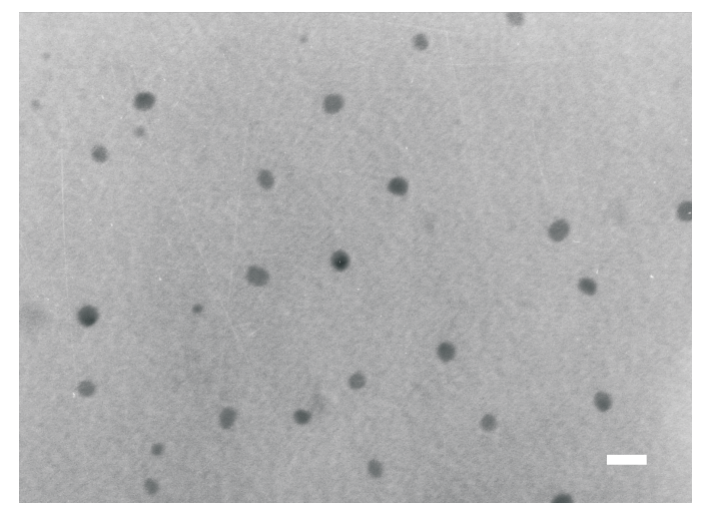

Fig. 3. Transmission microscopy of the m-AuNPs. Scale bar $=20 \mathrm{~nm}$.

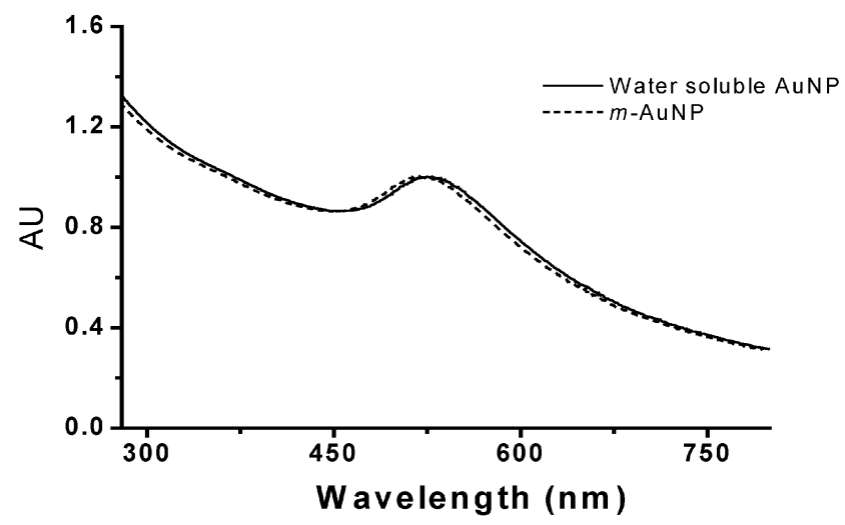

Fig. 4. Typical UV-Vis spectrum of the water soluble AuNPs and $m$-AuNPs. 
after modification also indicated that no aggregation occurred in aqueous media. ${ }^{10}$ The observed plasmon band at $520 \mathrm{~nm}$ also suggests that the sizes of gold nanoparticles are indeed within the nanometer region. As estimated from the average nanoparticle diameter and the result of elemental analysis, a single $m$-AuNP consists of approximately 200 attached carbohydrates. ${ }^{11}$

\section{Stability of Carbohydrate-Gold Nanoparticles}

Several reports have shown previously that gold nanoparticles stabilized by surfactants or polymers were not effective in preventing aggregation of the nanoparticles, particularly under high concentrations of salt medium. ${ }^{12}$ The stability of carbohydrate-AuNPs in various biological media was further examined before its application as a contrast agent for OA imaging. Based on the results of their UV-vis absorption measurements, $m$-AuNPs were found to be very stable in deionized water and buffers of elevated salt concentrations, and its stability was independent of high ion strength and $\mathrm{pH}$ values in the range from 1.5 to 12 of solutions (Fig. 5). The extraordinary diversity of carbohydrate-AuNPs allows gold nanoparticles to be easily redissolved in aqueous media without aggregation. These properties have made application of carbohydrate-AuNPs in biological systems feasible.

\section{Hemocompatibility Measurements}

The in vitro hemolysis assay was performed by the detection of free hemoglobin in the serum released from the disruptured red blood cells after incubation with various concentrations of the nanoparticles. As shown in Fig. 7, the

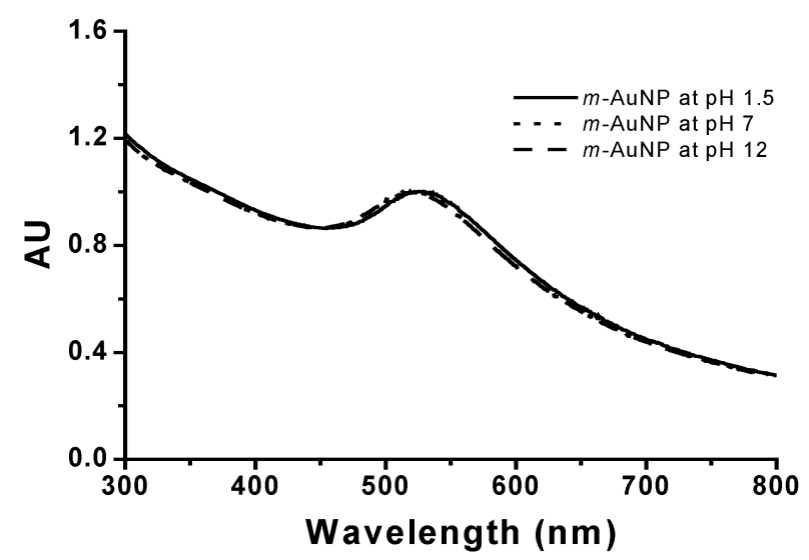

Fig. 5. The extreme results of the stability measurements for $m$-AuNP, the following $\mathrm{pH}$ values $1.5,7,12$ of UV-vis absorption measurements are shown. results indicated that significant hemolysis $(0.2 \mathrm{~g} / \mathrm{dL})$ could only be detected when the nanoparticle concentration was above $4.85 \times 10^{-9} \mathrm{M}$, which is 8 times higher than that required for the imaging purposes according to this study. The $m$-AuNPs were therefore further evaluated for their imaging and blood flow estimation in the following study.

Blood flow measurement provides critical clinical information for disease diagnosis and treatment in a wide range of clinical diseases. Blood flow and oxygenation of human tumors are important for their resistance to radiation therapy and chemotherapy that leads to a final clinical treatment failure. ${ }^{13}$ Increase in the tumor blood supply has been reported to be associated with regional lymph node metastasis. ${ }^{14}$ The measurement and imaging of blood flow also play important roles in the diagnosis of coronary artery disease, arthrosclerosis, hepatic function, erectile dysfunction, etc. ${ }^{15,16}$

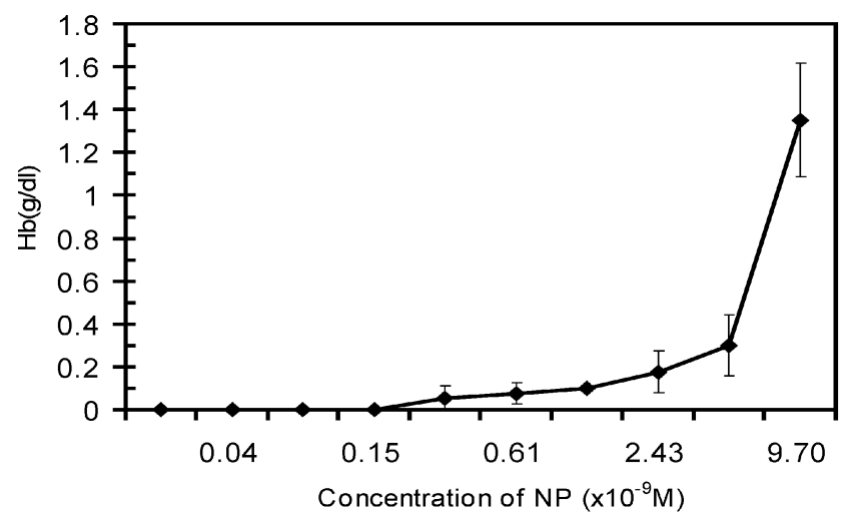

Fig. 6. Hemolysis assay of the $m$-Au NP in human whole blood.

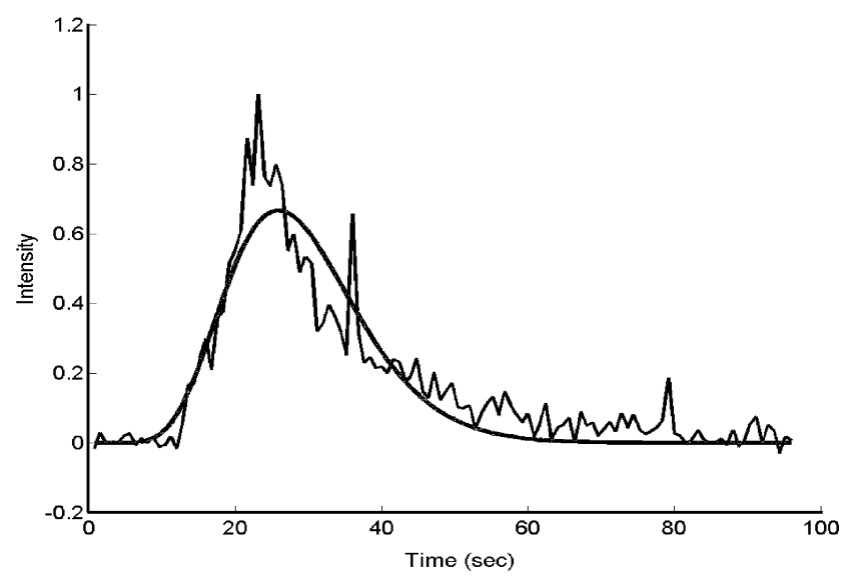

Fig. 7. A typical TIC (dashed) and its fitted curve to a gamma function (solid). 
Current methods for blood flow measurement are mostly based on ultrasonic or laser Doppler imaging. However, the use of both methods only gives a semi-quantitative index of the tissue perfusion. Although recent technological advancement has allowed the analysis of both volumetric and velocimetric components, the accuracy and sensitivity of the measurements still demand further improvement. Recent advancement of OA imaging techniques has enabled better dynamic and quantitative measurements of tissues. However, only a few have reported the applications of nanoparticle-based contrast agents.

Gold nanoparticles have been investigated for both in vitro and in vivo biomedical applications due to their biocompatibility, stability and mature crosslinking strategies for biomolecules. ${ }^{17}$ Nanoparticles with gold cores or shells have been used as targeted drug carriers and photonic based diagnosis and therapy of solid tumors. ${ }^{17 \mathrm{a}, 18}$ Gold nanoparticles for OA blood flow measurements have not been reported.

\section{Opto-Acoustic TIC Measurements}

Perfusion is defined as the flow per unit volume. ${ }^{19}$ When a contrast agent is injected, the perfusion can be calculated by dividing the fractional vascular volume by the mean transit time (MTT). In other words, if the TIC and vascular volume in the region of interest can be measured, perfusion can be calculated. Since perfusion represents blood flow normalized to the quantity of tissue supplied, it is clearly a very important measure of microcirculation. Considering a simple perfused area, blood flow at a rate $(Q)$ enters and leaves a perfusion model of volume $(V)$ via a single input vessel and a single output vessel. The concentration of contrast agent entering the model (i.e., the number of microbubbles per unit volume) as a function of time (i.e., the input function) is defined as $n_{I}(t)$. According to the dilution theory, the concentration of the contrast agent at the output end of the chamber $n_{o}(t)$ (i.e., the output function) is given by

$$
n_{o}(t)=n_{I}(t) \otimes h(t)
$$

where $\otimes$ stands for convolution, and $h(t)$ is the transfer function of the perfusion model. Note that the measured intensity is assumed linearly related to the concentration; this assumption has also been made in other studies. ${ }^{7 \mathrm{a}, 7 \mathrm{c}, 8,20}$

The time constant $\tau$, which can be considered as the system's transit time, is defined as the ratio of the mixing volume to the volume flow rate, i.e., $\tau=V / Q$. In this case, the transfer function is given by ${ }^{21}$

$$
h(t)=\left\{\begin{array}{cc}
0 & t<0 \\
\frac{1}{\tau} e^{-t / \tau} & t>0
\end{array} .\right.
$$

In addition to $\tau$, the TIC is used to derive other parameters in this study, including MTT. The MTT is defined as

$$
M T T=\frac{\int_{0}^{\alpha} t f(t) d t}{\int_{0}^{\alpha} f(t)},
$$

where $f(t)$ is the measured intensity as a function of time $t$. The MTT represents the time for the entire fluid volume to pass though the perfused area. According to the indicator-dilution theory, there is an inverse relation between the flow rate and the MTT. If the transfer function is defined as in (2), it can be shown that

$$
M T T=\tau=\frac{V}{Q},
$$

To reduce estimation errors in the time-intensity measurements, the TIC is fitted to a gamma function $g(t)$ defined by

$$
g(t)=\alpha\left(t-t_{o}\right)^{\gamma} e^{-\beta\left(t-t_{o}\right)}
$$

where $t_{0}$ indicates a time delay, $\alpha$ and $\beta$ are scaling factors, and $\gamma$ represents the skewness. The gamma function is used to represent a wide range of characteristics of possible TIC curves. ${ }^{21}$ Certainly, validity of the gamma-function fitting may be questionable if the flow model cannot be adequately described by the indicator dilution theory.

The dashed line in Fig. 7 shows a typical measured TIC. The curve after gamma function fitting is shown as the solid line. Note that the wash-out phase of the TIC generally can be fitted to an exponential function. The true flow rate was $72 \mathrm{mi} / \mathrm{min}$, and the theoretical time constant is 25 sec, versus the measured $29 \mathrm{sec}$ of the MTT.

\section{CONCLUSIONS}

In this study, we have prepared different types of carbohydrate-AuNPs for use as OA contrast agents in a biological system. The experimental results indicated that the conjugates are stable and biocompatible in various biological media solutions. The hemocompatibility of the as-synthesized gold nano-contrast agent in the test dosage range 
was demonstrated and encouraged our future evaluation of an in vivo model system. We successfully demonstrated the feasibility of using carbohydrate-AuNPs as the OA contrast agent for indicator-dilution based blood flow measurements. Another future project will include the correlation between the derived parameters (e.g., MTT) and the flow parameters (e.g., $V$ and $Q$ ). The sensitivity will also be evaluated to improve the properties of the nanoparticlebased image contrast and the detection mechanism for in vivo applications.

\section{ACKNOWLEDGMENTS}

The authors acknowledge financial support from the NSC, NNSTP, IAMS (Academia Sinica), Ministry of Education, NHRI and NTNU. The authors also thank Liao, C. $\mathrm{K}$. for helpful discussion and setting up the experiments.

Received June 17, 2007.

\section{REFERENCES}

1. For recent review, see: Niemeyer, C. M. Angew. Chem., Int. Ed. 2001, 40, 4128.

2. (a) Hayat, M. A. Colloid Gold: Principles, Methods and Applications; Academic Press: New York, 1989. (b) Kreuter, J. In Microcapsules and Nanoparticles in Medicine and Pharmacy; Donbrow, M., Ed.; CRC: Boca Raton, 1992.

3. (a) Alivisatos, A. P.; Peng, X.; Wilson, T. E.; Johnsson, K. P.; Loweth, C. J.; Bruchez, M. P.; Jr., Schultz, P. G. Nature 1996, 382, 609. (b) Mirkin, C. A.; Letsinger, R. L.; Mucic, R. C.; Storhoff, J. J. Nature 1996, 382, 607. (c) Taton, T. A.; Lu, G.; Mirkin, C. A. J. Am. Chem. Soc. 2001, 123, 5164. (d) Taton, T. A.; Mirkin, C. A.; Letsinger, R. L. Science 2000, 289,1757

4. (a) Boal, A. K.; Ilhan, F.; DeRouchey, J. E.; Thurn-Albrecht, T.; Russell, T. P.; Rotello, V. M. Nature 2000, 404, 746. (b) Boal, A. K.; Rotello, V. M. J. Am. Chem. Soc. 2000, 122, 734.

5. Lin, C. C.; Yeh, Y. C.; Yang, C. Y.; Chen, C. L.; Chen, G. F.; Chen, C. C.; Wu, Y. C. J. Am. Chem. Soc. 2002, 124, 3508.
6. Lin, C. C.; Yeh, Y. C.; Yang, C. Y.; Chen, G. F.; Chen, Y. C.; Wu, Y. C.; Chen, C. C. Chem. Commun. 2003, 23, 2920.

7. (a) Li, P. C.; Yeh, C. K.; Wang, S. W. Ultrasound Med. Biol. 2002, 28, 349. (b) Ugolini, P., Delouche, A., Herment, A., Diebold, B. Ultrasound Med. Biol. 2000, 26, 113. (c) Yeh, C. K.; Wang, S. W.; Li, P. C. Ultrason. Imaging 2001, 23, 90.

8. Mor-Avi, V.; Akselrod, S.; David, D.; Keselbrener, L.; Bitton, Y. Ultrasound Med. Biol. 1993, 19, 635.

9. Keating, C. D.; Kovaleski, K. M.; Natan, M. J. J. Phys. Chem. B 1998, 102, 9404, and reference therein.

10. Storhoff, J. J.; Lazarides, A. A.; Mucic, R. C.; Mirkin, C. A.; Letsinger, R. L.; Schatz, G. C. J. Am. Chem. Soc. 2000, 122, 4640.

11. Heister, K.; Zharnikov, M.; Grunze, M.; Johansson, L. S. O. J. Phys. Chem. B 2001, 105, 4058.

12. Sato, T.; Ruth, R. Stabilization of Colloidal Dispersions by Polymer Adsorption; Surfactant Science Series, No. 9; Marcel Dekker: New York, 1980; pp 65-119.

13. Feldmann, H. J.; Molls, M.; Vaupel, P. Strahlenther Onkol 1999, 175,1 .

14. Nathanson, S. D. Cancer 2003, 98, 413.

15. (a) Bishop, A. H.; Samady, H. Am. Heart J. 2004, 147, 792. (b) Bisoendial, R. J.; Hovingh, G. K.; de. Groot, E.; Kastelein, J. J.; Lansberg, P. J.; Stroes, E. S. Curr. Opin. Lipidol 2002, 13, 595 .

16. (a) Aversa, A.; Caprio, M.; Spera, G..; Fabbri, A. J. Endocrinol. Invest. 2003, 26, 122. (b) Chow, P. K.; Yu, W. K.; Soo, K. C; Chan, S. T. J. Surg. Res. 2003, 112, 1.

17. (a) Loo, C.; Lin, A.; Hirsch, L.; Lee, M. H.; Barton, J.; Halas, N.; West, J.; Drezek, R. Technol. Cancer Res. Treat 2004, 3, 33. (b) Nath, N.; Chilkoti, A. Anal. Chem. 2002, 74, 504.

18. Paciotti, G. F.; Myer, L.; Weinreich, D.; Goia, D.; Pavel, N.; McLaughlin, R. E.; Tamarkin, L. Drug Deliv. 2004, 11, 169.

19. Nanda, N. C.; Schlief, R.; Goldberg, B. Advances in Echo Imaging Using Contrast Enhancement. Dordrecht; Boston: Kluwer Academic Publishers, 1997.

20. Heidenreich, P. A.; Wiencek, J. G.; Zaroff, J. G. et al. J. Am. Soc. Echocardiogr. 1993, 6, 51.

21. Chen, X.; Schwarz, K. Q.; Phillips, D.; Steinmetz, S. D.; Schlief, R. A. IEEE Trans. Biomed. Eng. 1998, 45, 754.

22. Oraevsky, A. A.; Jacques, S. L.; Esenaliev, R. O.; Tittel, F. K. Laser-based Optoacoustic Imaging in Biological Tissues; Proc. SPIE 1994; 2134A; pp 122-128. 